PROCEEDINGS OF THE

AMERICAN MATHEMATICAL SOCIETY

Volume 132, Number 10, Pages 2817-2824

S 0002-9939(04)07133-3

Article electronically published on June 2, 2004

\title{
BOUNDS OF GAUSS SUMS IN FINITE FIELDS
}

\author{
IGOR E. SHPARLINSKI
}

(Communicated by Wen-Ching Winnie Li)

Abstract. We consider Gauss sums of the form

$$
G_{n}(a)=\sum_{x \in \mathbb{F}_{p} m} \chi\left(x^{n}\right)
$$

with a nontrivial additive character $\chi \neq \chi_{0}$ of a finite field $\mathbb{F}_{p} m$ of $p^{m}$ elements of characteristic $p$. The classical bound $\left|G_{n}(a)\right| \leq(n-1) p^{m / 2}$ becomes trivial for $n \geq p^{m / 2}+1$. We show that, combining some recent bounds of HeathBrown and Konyagin with several bounds due to Deligne, Katz, and Li, one can obtain the bound on $\left|G_{n}(a)\right|$ which is nontrivial for the values of $n$ of order up to $p^{m / 2+1 / 6}$. We also show that for almost all primes one can obtain a bound which is nontrivial for the values of $n$ of order up to $p^{m / 2+1 / 2}$.

\section{INTRODUCTION}

Let $p$ be a prime and let $\mathbb{F}_{p^{m}}$ be a finite field of $p^{m}$ elements of characteristic $p$. We recall that the function

$$
\operatorname{Tr}(z)=\sum_{i=0}^{m-1} z^{p^{i}}
$$

is called the trace and the function

$$
\operatorname{Nm}(z)=\prod_{i=0}^{m-1} z^{p^{i}}
$$

is called the norm of $z \in \mathbb{F}_{p^{m}}$ in $\mathbb{F}_{p}$.

We define $\mathbf{e}_{p}(z)=\exp (2 \pi i / p)$. Then the set of functions $\chi_{a}(z)=\mathbf{e}_{p}(\operatorname{Tr}(a z))$, $a \in \mathbb{F}_{p^{m}}$, form the set of additive characters of $\mathbb{F}_{p^{m}}$, with $\chi_{0}$ being the trivial character.

Let us define the Gauss sums

$$
G_{n}(a)=\sum_{x \in \mathbb{F}_{p^{m}}} \mathbf{e}_{p}\left(\operatorname{Tr}\left(a x^{n}\right)\right) .
$$

It is easy to see that

$$
G_{n}(a)=G_{d}(a)
$$

where $d=\operatorname{gcd}\left(n, p^{m}-1\right)$, thus throughout the paper we always assume that $n \mid p^{m}-1$.

Received by the editors February 1, 2002 and, in revised form, June 7, 2002.

2000 Mathematics Subject Classification. Primary 11L05, 11T24; Secondary 11B37.

Key words and phrases. Gauss sums, finite fields, linear recurrence sequences. 
The classical result which can be found in [11] and in many other sources says that

$$
\max _{a \in \mathbb{F}_{p}^{*}}\left|G_{n}(a)\right| \leq(n-1) p^{m / 2} .
$$

The bound is nontrivial for $n \leq p^{m / 2-\delta}$ with some fixed $\delta>0$. For the important special case of $m=1$, Heath-Brown and Konyagin [5] obtained a bound which is nontrivial for values of $n$ of order up to $p^{2 / 3-\delta}$; see also Theorem 3.4 of $[8$. Konyagin [7] has recently improved this result and obtained a nontrivial bound for $n$ up to $p^{3 / 4-\delta}$ (also for $m=1$ ). Unfortunately neither the method of [7] nor of [5] nor of the previous improvement of (1.1) from [12, 13] can be extended to the case $m>1$. However here we show that combining these results with the bounds of some other exponential sums introduced and studied by Deligne [2] and then by Katz [6] and Li 9, 10], one can obtain an improvement of (1.11) for an arbitrary $m \geq 1$. To be more precise, our result improves (1.1) for $n$ close to $p^{m / 2}$ and in fact provides a nontrivial estimate beyond this threshold for values of $n$ up to $p^{m / 2+1 / 6-\delta}$. Moreover we show that by using Theorem 5.5 of $[8$, for almost all primes one can obtain an even slightly stronger bound, which, for every fixed $m \geq 1$, remains nontrivial for values of $n$ up to $p^{m / 2+1 / 2-\delta}$.

In fact it is technically more convenient to work with the sums

$$
S_{t}(a)=\sum_{z=1}^{t} \mathbf{e}_{p}\left(\operatorname{Tr}\left(a \vartheta^{z}\right)\right)
$$

where $\vartheta \in \mathbb{F}_{p^{m}}^{*}$ is an element of multiplicative order $t$ (it is easy to see that $S_{t}(a)$ does not depend on the choice of $\vartheta$ ).

One easily verifies that, for $t=\left(p^{m}-1\right) / n$,

$$
G_{n}(a)=n S_{t}(a)+1 .
$$

It is also well known (see Chapter 5 of [8]) that

$$
\max _{a \in \mathbb{F}_{p}^{*}}\left|S_{t}(a)\right| \leq p^{m / 2}
$$

which is nontrivial for $t \geq p^{m / 2+\delta}$ with some fixed $\delta>0$.

We improve this bound provided that the part of the multiplicative group generated by $\vartheta$, which does not belong to any proper subfield of $\mathbb{F}_{p^{m}}$, is not too small. This can be expressed as the inequality on

$$
\max _{\substack{1 \leq \nu<m \\ \nu \mid m}} \operatorname{gcd}\left(p^{\nu}-1, t\right) \leq t p^{-\rho}
$$

which we assume to hold for some fixed $\rho>0$. For $n=\left(p^{m}-1\right) / t$ this condition is equivalent to

$$
\operatorname{gcd}\left(\frac{p^{m}-1}{p^{\nu}-1}, n\right) \leq p^{-\rho} \frac{p^{m}-1}{p^{\nu}-1}, \quad 1 \leq \nu<m, \nu \mid m .
$$

The conditions (1.4) and (1.5) mean that a substantial portion of the cyclic group generated by $\vartheta$ and the group of nonzero $n$th powers, respectively, does not belong to any proper subfield of $\mathbb{F}_{p^{m}}$.

It is easy to show that (1.4), or another similar condition is necessary for a nontrivial upper bound on $S_{t}(a)$ (we remark that for $t \geq p^{m / 2+\delta}$ it is automatic). 
Indeed, it is easy to see that if $m=2 r$ is even and $t=p^{r}-1$, then for any $a \in \mathbb{F}_{p^{m}}^{*}$ with

we have

$$
a^{p^{r}-1}=-1
$$

thus

$$
a^{p^{i+r}} \vartheta^{x p^{i+r}}=(-a)^{p^{i}} \vartheta^{x p^{i}}=-a^{p^{i}} \vartheta^{x p^{i}}
$$

$$
\operatorname{Tr}\left(a \vartheta^{x}\right)=\sum_{i=0}^{m-1}\left(a \vartheta^{x}\right)^{p^{i}}=\sum_{i=0}^{r-1}\left(a^{p^{i}} \vartheta^{x p^{i}}+a^{p^{i+r}} \vartheta^{x p^{i+r}}\right)=0 .
$$

Therefore $S_{t}(a)=t$ for such $t$ and $a$.

Throughout the paper the implied constants in symbols ' $O$ ' and ' $\ll$ ' are absolute and can be efficiently evaluated (we recall that $A \ll B$ is equivalent to $A=O(B)$ ).

\section{Preparations}

As we have mentioned we need some results corresponding to the case $m=1$. The following statement has been proved in [5]; see also Theorem 3.4 of [8].

Lemma 2.1. Let $g \in \mathbb{F}_{p}^{*}$ be of multiplicative order $\tau$ modulo $p$. Then the bound

$$
\max _{\operatorname{gcd}(c, p)=1}\left|\sum_{z=1}^{\tau} \mathbf{e}_{p}\left(c g^{z}\right)\right| \ll B(\tau, p)
$$

holds, where

$$
B(\tau, p)=\min \left\{p^{1 / 2}, p^{1 / 4} \tau^{3 / 8}, p^{1 / 8} \tau^{5 / 8}\right\} .
$$

The following statement follows from the stronger and more explicit result of [7].

Lemma 2.2. For any $\delta>0$, there exists $\eta>0$ such that for any $g \in \mathbb{F}_{p}^{*}$ of multiplicative order $\tau \geq p^{1 / 4+\eta}$ modulo $p$, the bound

$$
\max _{\operatorname{gcd}(c, p)=1}\left|\sum_{z=1}^{\tau} \mathbf{e}_{p}\left(c g^{z}\right)\right| \ll \tau p^{-\eta}
$$

holds.

We also need the estimate which follows from Theorem 5.5 of 8 ]; see also [4].

Lemma 2.3. Let $Q$ be a sufficiently large integer. Then, for any $\delta>0$, there exists $\eta>0$ such that for all primes $p \in[Q, 2 Q]$ except at most $Q^{3 / 4+\delta}$ of them, for any $g \in \mathbb{F}_{p}$ of multiplicative order $\tau \geq p^{\delta}$ the bound

$$
\max _{\operatorname{gcd}(c, p)=1}\left|\sum_{x=0}^{\tau-1} \mathbf{e}_{p}\left(c g^{x}\right)\right|=O\left(\tau p^{-\eta}\right)
$$

holds.

Proof. For each integer $\tau \geq 1$ and for each prime $p \equiv 1(\bmod \tau)$ we fix an element $g_{p, \tau}$ of multiplicative order $\tau$. Then Theorem 5.5 of [8] claims that for any $U>1$ and any integer $\nu \geq 2$, for all primes $p \equiv 1(\bmod \tau)$ except at most $O(U / \log U)$ of them, the bound

$$
\max _{\operatorname{gcd}(c, p)=1}\left|\sum_{x=0}^{\tau-1} \mathbf{e}_{p}\left(c g_{p, \tau}^{x}\right)\right|=O\left(\tau p^{1 / 2 \nu^{2}}\left(\tau^{-1 / \nu}+U^{-1 / \nu^{2}}\right)\right)
$$


holds. We remark that the value of the above exponential sum does not depend on the particular choice of the element $g_{p, \tau}$.

Taking

$$
\nu=\left\lfloor\frac{1}{\delta}\right\rfloor+1 \quad \text { and } \quad U=Q^{1 / 2+\delta / 3},
$$

after simple computation we obtain that there exists some $\eta>0$, depending only on $\delta$, such that for any fixed $\tau \geq Q^{\delta}$ the bound

$$
\max _{\operatorname{gcd}(c, p)=1}\left|\sum_{x=0}^{\tau-1} \mathbf{e}_{p}\left(c g_{p, \tau}^{x}\right)\right| \leq \tau p^{-\eta}
$$

holds for all except $O\left(Q^{1 / 2+\delta / 3}\right)$ primes $p \equiv 1(\bmod \tau)$ in the interval $p \in[Q, 2 Q]$. As it follows from Lemma [2.2, a similar bound also holds for $\tau \geq Q^{1 / 4+\delta / 3}$. So the total number of exceptional primes $p$ for which the bound of the lemma does not hold for at least one $\tau \geq p^{\delta} \geq Q^{\delta}$ is $O\left(Q^{3 / 4+2 \delta / 3}\right)$. Thus for sufficiently large $Q$ we obtain the desired result.

Let us denote by $\mathcal{N}$ the set of elements $z \in \mathbb{F}_{p^{m}}$ with $\operatorname{Nm}(z)=1$. Thus

$$
|\mathcal{N}|=\frac{p^{m}-1}{p-1} .
$$

Our results depend on the following estimate conjectured by Deligne in [2] (and proved in the case of the trivial character $\chi=\chi_{0}$ ). Then in some special cases it is also given in [9] and in Chapter 6 of [10]. In the full generality it has been proved by Katz [6] (the proof follows from Theorem 4.1.1 of [6] after some standard transformations).

Lemma 2.4. For any $\gamma \in \mathbb{F}_{p^{m}}^{*}$ and any multiplicative character $\chi$ of $\mathbb{F}_{p^{m}}^{*}$, the bound

$$
\left|\sum_{u \in \mathcal{N}} \chi(u) \mathbf{e}_{p}(\operatorname{Tr}(\gamma u))\right| \leq m p^{(m-1) / 2}
$$

holds.

We recall the following standard property of the group of characters of an abelian group.

Lemma 2.5. Let $\mathcal{H}$ be an abelian group and let $\widehat{\mathcal{H}}=\operatorname{Hom}\left(\mathcal{H}, \mathbb{C}^{*}\right)$ be its dual group. Then for any $h \in \mathcal{H}$, we have

$$
\frac{1}{|\widehat{\mathcal{H}}|} \sum_{\chi \in \widehat{\mathcal{H}}} \chi(h)= \begin{cases}1, & \text { if } h=\mathrm{id}, \\ 0, & \text { if } h \neq \mathrm{id},\end{cases}
$$

where id is the identity element of $\mathcal{H}$.

Lemma 2.6. For any subgroup $\mathcal{G}$ of $\mathcal{N}$ and any $\gamma \in \mathbb{F}_{p^{m}}^{*}$, the bound

$$
\left|\sum_{u \in \mathcal{G}} \mathbf{e}_{p}(\operatorname{Tr}(\gamma u))\right| \leq m p^{(m-1) / 2}
$$

holds. 
Proof. Let $\Omega_{\mathcal{G}}$ be the set of all multiplicative characters of $\mathcal{N}$ trivial on $\mathcal{G}$. Using Lemma 2.5] we write

$$
\begin{aligned}
\sum_{u \in \mathcal{G}} \mathbf{e}_{p}(\operatorname{Tr}(\gamma u)) & =\frac{1}{\left|\Omega_{\mathcal{G}}\right|} \sum_{u \in \mathcal{N}} \mathbf{e}_{p}(\operatorname{Tr}(\gamma u)) \sum_{\chi \in \Omega_{\mathcal{G}}} \chi(u) \\
& =\frac{1}{\left|\Omega_{\mathcal{G}}\right|} \sum_{\chi \in \Omega_{\mathcal{G}}} \sum_{u \in \mathcal{N}} \chi(u) \mathbf{e}_{p}(\operatorname{Tr}(\gamma u)) .
\end{aligned}
$$

Applying the inequality of Lemma 2.4 we obtain the desired estimate.

The following bound on the number of zeros of sparse polynomials is a version of a similar result from [1, 3]. We present it in the form given in [12].

Lemma 2.7. Let $\vartheta \in \mathbb{F}_{p^{m}}$ be of multiplicative order $t$ and let $s \geq 2$ be an integer. For elements $a_{1}, \ldots, a_{s} \in \mathbb{F}_{p^{m}}^{*}$ and $s$ integers $e_{1}, \ldots, e_{s}$ we denote by $W$ the number of solutions of the equation

$$
\sum_{i=1}^{s} a_{i} \vartheta^{e_{i} u}=0, \quad u \in[0, t-1]
$$

Then

$$
W \leq 3 t^{1-1 /(s-1)} D^{1 /(s-1)},
$$

where

$$
D=\min _{1 \leq i \leq s} \max _{j \neq i} \operatorname{gcd}\left(e_{j}-e_{i}, t\right) .
$$

From Lemma 2.7 one immediately derives an upper bound on the number $N_{a}$ of zero values of $\operatorname{Tr}\left(a \vartheta^{x}\right), x=0, \ldots, t-1$.

Lemma 2.8. Let $m \geq 2$ and let $\vartheta \in \mathbb{F}_{p^{m}}^{*}$ be of multiplicative order $t$. Then for any $a \in \mathbb{F}_{p^{m}}^{*}$

$$
N_{a} \leq 3 t^{1-1 /(m-1)} d^{1 /(m-1)},
$$

where

$$
d=\max _{\substack{1 \leq \nu<m \\ \nu \mid m}} \operatorname{gcd}\left(p^{\nu}-1, t\right)
$$

Proof. The equation $\operatorname{Tr}\left(a \vartheta^{x}\right)=0$ is equivalent to the equation

$$
\sum_{i=0}^{m-1} a^{p^{i}} \vartheta^{x p^{i}}=0, \quad x=0, \ldots, t-1 .
$$

We recall that

$$
\operatorname{gcd}\left(p^{k}-1, p^{m}-1\right)=p^{\operatorname{gcd}(k, m)}-1 .
$$

Because $t$ is a divisor of $p^{m}-1$ we see that $\operatorname{gcd}(p, t)=1$ and from Lemma [2.7] we derive the result. 


\section{MAin Results}

Here we obtain an improvement of (1.3) which holds for all primes $p$.

Theorem 3.1. For any $m \geq 2$ and $t \mid p^{m}-1$ satisfying (1.4), the bound

$$
\max _{a \in \mathbb{F}_{p}^{*}}\left|S_{t}(a)\right| \ll t p^{-\rho /(m-1)}+m^{3 / 11} t^{8 / 11} p^{(3 m-1) / 22}
$$

holds.

Proof. We define

$$
\tau=\operatorname{gcd}(t, p-1) \quad \text { and } \quad T=t / \tau .
$$

Let $\vartheta \in \mathbb{F}_{p^{m}}^{*}$ be an element of multiplicative order $t$. Then

$$
S_{t}(a)=\sum_{z=1}^{t} \mathbf{e}_{p}\left(\operatorname{Tr}\left(a \vartheta^{z}\right)\right)=\frac{1}{\tau} \sum_{z=1}^{t} \sum_{u=1}^{\tau} \mathbf{e}_{p}\left(\operatorname{Tr}\left(a \vartheta^{T u+z}\right)\right) .
$$

Let $g=\vartheta^{T}$. It is clear that $g \in \mathbb{F}_{p}$ (because $g^{p-1}=\vartheta^{T(p-1)}=1$ ) and that $g$ is of multiplicative order $\tau$. Therefore,

$$
S_{t}(a)=\frac{1}{\tau} \sum_{z=1}^{t} \sum_{u=1}^{\tau} \mathbf{e}_{p}\left(g^{u} \operatorname{Tr}\left(a \vartheta^{z}\right)\right) .
$$

For $N_{a}$ values of $z=1, \ldots, t$ with $\operatorname{Tr}\left(a \vartheta^{z}\right)=0$ the inner sum is equal to $\tau$, otherwise the bound of Lemma 2.1 applies. Together with Lemma 2.8 it gives

$$
\left|S_{t}(a)\right| \ll N_{a}+t p^{1 / 8} \tau^{-3 / 8} \ll t p^{-\rho /(m-1)}+t p^{1 / 8} \tau^{-3 / 8} .
$$

Similarly,

$$
S_{t}(a)=\sum_{z=1}^{t} \mathbf{e}_{p}\left(\operatorname{Tr}\left(a \vartheta^{z}\right)\right)=\frac{1}{T} \sum_{z=1}^{t} \sum_{v=1}^{T} \mathbf{e}_{p}\left(\operatorname{Tr}\left(a \vartheta^{\tau v+z}\right)\right) .
$$

Let $\lambda=\vartheta^{\tau}$. Then

$$
S_{t}(a)=\frac{1}{T} \sum_{z=1}^{t} \sum_{v=1}^{T} \mathbf{e}_{p}\left(\operatorname{Tr}\left(a \vartheta^{z} \lambda^{v}\right)\right) .
$$

It is clear that $\lambda$ is of multiplicative order $T$. We also have

$$
T \mid \frac{p^{m}-1}{\tau} \quad \text { and } \quad \operatorname{gcd}(T,(p-1) / \tau)=1 .
$$

Therefore

$$
T \mid \frac{p^{m}-1}{p-1},
$$

thus

$$
\operatorname{Nm}(\lambda)=\lambda^{\left(p^{m}-1\right) /(p-1)}=1 .
$$

Hence from Lemma 2.6 we obtain the bound

$$
\left|S_{t}(a)\right| \leq m t T^{-1} p^{(m-1) / 2}=m \tau p^{(m-1) / 2} .
$$

Using the bound (3.1) for

$$
\tau \geq t^{8 / 11} p^{-(4 m-5) / 11} m^{-8 / 11}
$$

and the bound (3.2) otherwise, we obtain the desired result. 
Assuming that $m$ is fixed, we see that the bound of Theorem 3.1 is nontrivial for $t \geq p^{m / 2-1 / 6+\delta}$ for any fixed $\delta>0$.

Now we show that for almost all primes a slightly stronger bound holds which is nontrivial for $t \geq p^{m / 2-1 / 2+\delta}$.

Theorem 3.2. Let $Q$ be a sufficiently large integer. Then, for any $\delta>0$, there exists some $\gamma>0$ such that for all primes $p \in[Q, 2 Q]$ except at most $Q^{3 / 4+\delta}$ of them, such that for any $m \geq 2$ and $t \mid p^{m}-1$ satisfying (1.4) and $t \geq p^{(m-1) / 2+\delta}$, the bound

holds.

$$
\max _{a \in \mathbb{F}_{p^{m}}^{*}}\left|S_{t}(a)\right| \ll t p^{-\rho /(m-1)}+t p^{-\gamma}
$$

Proof. As in the proof of Theorem 3.1 we define

$$
\tau=\operatorname{gcd}(t, p-1) \quad \text { and } \quad T=t / \tau .
$$

Then from Lemma 2.8 and Lemma 2.3 we derive that the bound

$$
\left|S_{t}(a)\right| \ll t p^{-\rho /(m-1)}+t p^{-\eta}
$$

holds for all for all primes $p \in[Q, 2 Q]$ except at most $Q^{3 / 4+\delta / 2}$ of them, provided that $\tau>p^{\delta / 2}$. On the other hand, if $\tau \leq p^{\delta / 2}$, then $T \geq p^{(m-1) / 2+\delta / 2}$ and from (3.2) we derive

$$
\left|S_{t}(a)\right| \leq m t p^{-\delta / 2}
$$

Taking into account that for $m \geq \log p$ the bound is trivial, from (3.3) and (3.4) the desired result follows.

Applying (1.2), one immediately obtains the corresponding statements for $G_{n}(a)$.

Theorem 3.3. For any $m \geq 2$ and $n \mid p^{m}-1$ satisfying (1.5), the bound

$$
\max _{a \in \mathbb{F}_{p}^{*}}\left|G_{n}(a)\right| \ll p^{m-\rho /(m-1)}+m^{3 / 11} n^{3 / 11} p^{m-(3 m+1) / 22}
$$

holds.

Theorem 3.4. Let $Q$ be a sufficiently large integer. Then, for any $\delta>0$, there exists some $\gamma>0$ such that for all primes $p \in[Q, 2 Q]$ except at most $Q^{3 / 4+\delta}$ of them, such that for any $m \geq 2$ and $n \mid p^{m}-1$, satisfying [1.5) and $n \leq p^{(m+1) / 2-\delta}$, the bound

holds.

$$
\max _{a \in \mathbb{F}_{p}^{*}}\left|G_{n}(a)\right| \ll p^{m-\rho /(m-1)}+p^{m-\gamma}
$$

\section{Concluding Remarks}

It is well known that any linear recurrence sequence $u(x) \in \mathbb{F}_{p}$ of order $m \geq 2$, satisfying an equation

$$
u(x+m)=c_{m-1} u(x+m-1)+\ldots+c_{0} u(x), \quad x=1,2, \ldots,
$$

with an irreducible polynomial

$$
f(X)=X^{m}-c_{m-1} X^{m-1}-\ldots-c_{0} \in \mathbb{F}_{p}[X],
$$

can be uniquely represented as

$$
u(x)=\operatorname{Tr}\left(a \vartheta^{x}\right), \quad x=1,2, \ldots,
$$


where $a, \vartheta \in \mathbb{F}_{p^{m}}$, and $\vartheta$ is a root of $f(X)$; see Theorem 8.24 of [1]. Thus our results apply to exponential sums with such sequences, giving the first nontrivial bound in the case where the period of $u(x)$ is less than the $p^{m / 2}$ threshold; see Section 8.7 of [11] for an outline of previously known results.

We remark that in the proof of Theorem 3.1 we use only the last one out of the three upper bounds given in Lemma 2.1. It seems that using the other two bounds does not produce any further improvement.

Finally we note that if $m \geq 3$, then for $t \leq p^{m / 3}$ and $n \geq p^{2 m / 3}$ our results are trivial. Therefore, for any $m \geq 3$, in the range where our results are nontrivial, the conditions (1.4) and (1.5) may fail only for $\nu=m / 2$. Therefore, for odd $m$ our results always apply and for any even $m$ one can replace (1.4) and (1.5) with

$$
\operatorname{gcd}\left(p^{m / 2}-1, t\right) \leq t p^{-\rho} \quad \text { and } \quad \operatorname{gcd}\left(p^{m / 2}+1, n\right) \leq p^{-\rho}\left(p^{m / 2}+1\right)
$$

respectively.

\section{REFERENCES}

[1] R. Canetti, J. B. Friedlander, S. Konyagin, M. Larsen, D. Lieman and I. E. Shparlinski, 'On the statistical properties of Diffie-Hellman distributions', Israel J. Math., 120 (2000), 23-46. MR 2001k:11258

[2] P. Deligne, Cohomologie 'etale (SGA $4 \frac{1}{2}$ ), Lect. Notes in Math., Springer-Verlag, Berlin, 569 (1977). MR 57:3132

[3] J. B. Friedlander, M. Larsen, D. Lieman and I. E. Shparlinski, 'On correlation of binary M-sequences', Designs, Codes and Cryptography, 16 (1999), 249-256. MR 2000g:94024

[4] M. I. González Vasco and I. E. Shparlinski, 'On the security of Diffie-Hellman bits', Proc. Workshop on Cryptography and Computational Number Theory, Singapore 1999, Birkhäuser, 2001, 257-268.

[5] D. R. Heath-Brown and S. V. Konyagin, 'New bounds for Gauss Sums derived from $k$ th powers, and for Heilbronn's exponential sum', Quart. J. Math., 51 (2000), 221-235. MR 2001h:11106

[6] N. M. Katz, Gauss sums, Kloosterman sums, and monodromy groups, Ann. of Math. Studies, 116, Princeton Univ. Press, 1988. MR 91a:11028

[7] S. V. Konyagin, 'Bounds of exponential sums over subgroups and Gauss sums', Preprint, 2002, 1-25 (in Russian).

[8] S. V. Konyagin and I. E. Shparlinski, Character sums with exponential functions and their applications, Cambridge Univ. Press, Cambridge, 1999. MR 2000h:11089

[9] W.-C. W. Li, 'Character sums and abelian Ramanujan graphs', J. Number Theory, 41 (1992), 199-217. MR 93h:11092

[10] W.-C. W. Li, Number theory with applications, World Scientific, Singapore, 1996.

[11] R. Lidl and H. Niederreiter, Finite fields, Cambridge University Press, Cambridge, 1997. MR 97i:11115

[12] I. E. Shparlinski, 'On bounds of Gaussian sums', Matem. Zametki, 50 (1991), 122-130 (in Russian). MR 92m:11082

[13] I. E. Shparlinski, 'On Gaussian sums for finite fields and elliptic curves', Proc. 1st FrenchSoviet Workshop on Algebraic Coding., Paris, 1991, Lect. Notes in Computer Sci., 537 (1992), 5-15. MR 95c:11146

Department of Computing, Macquarie University, Sydney, New South Wales 2109, Australia

E-mail address: igor@ics.mq.edu.au 